\title{
Effect of family enteral nutrition on nutritional status in elderly patients with esophageal carcinoma after minimally invasive radical surgery: a randomized trial
}

\author{
Tiantian Chen ${ }^{1 *}$, Wei Jiang ${ }^{2 \#}$, Guangming $\mathrm{He}^{2}$ \\ ${ }^{1}$ Department of Thoracic Surgery, the 904th Hospital of the Joint Service Support Center of the Chinese People's Liberation Army, Wuxi, China; \\ ${ }^{2}$ Department of Thoracic Surgery, Taixing People's Hospital of Jiangsu Province, Taixing, China \\ Contributions: (I) Conception and design: T Chen, W Jiang; (II) Administrative support: T Chen; (III) Provision of study materials or patients: All \\ authors; (IV) Collection and assembly of data: All authors; (V) Data analysis and interpretation: T Chen, W Jiang; (VI) Manuscript writing: All \\ authors; (VII) Final approval of manuscript: All authors. \\ \#These authors contributed equally to this work. \\ Correspondence to: Tiantian Chen. Department of Thoracic Surgery, the 904th Hospital of the Joint Service Support Center of the Chinese People's \\ Liberation Army, 101 Xingyuan North Road, Liangxi District, Wuxi 214000, China. Email: chentiantian808@163.com.
}

\begin{abstract}
Background Postoperative patients with esophageal carcinoma (EC) are prone to malnutrition. Studies have shown that the incidence of malnutrition after EC surgery reaches $60-80 \%$, and deaths due to malnutrition account for about 22\%. Patients with EC need at least 3 months to establish a new dietary pattern after surgery, so short-term enteral nutrition is of great significance. The aim of the present study was to investigate the effects of family enteral nutrition (FEN) on nutritional status in elderly patients with EC after minimally invasive radical surgery (MIS).

Methods: A total of 106 elderly patients with EC, who had undergone MIS at the 904th Hospital of the Joint Service Support Center of the Chinese People's Liberation Army and Taixing People's Hospital from January 2017 to July 2019 were selected to participate in the present study and randomly divided into the observation group and control group. There were 53 cases in each group. Patients in the control group were given regular meals after they were discharged from hospital, and the observation group was given FEN support based on the intervention of the control group. Body mass index (BMI), nutritional risk screening, nutritional status, and the incidence of complications were compared between the two groups at discharge and 1 month after discharge.
\end{abstract}

Results: The BMI of the observation group was higher than that of the control group 1 month after discharge $(\mathrm{P}<0.05)$. The nutritional risk ratio of the observation group was lower than that of the control group 1 month after discharge $(\mathrm{P}<0.05)$. Hemoglobin, serum albumin, serum total protein, transferrin, and serum prealbumin of the observation group were higher than the control group 1 month after discharge $(\mathrm{P}<0.05)$. There was no significant difference in the incidence of total complications between the two groups $(\mathrm{P}>0.05)$.

Conclusions: FEN could improve the nutritional status of elderly patients with EC after MIS and reduce the risk of postoperative malnutrition and incidence of complications.

Trial registration: Chinese Clinical Trial Registry ChiCTR2100046121.

Keywords: Family enteral nutrition (FEN); esophageal carcinoma (EC); minimally invasive radical surgery (MIS); nutritional risk screening; nutritional status; elderly patient

Submitted Apr 20, 2021. Accepted for publication Jun 15, 2021.

doi: 10.21037/apm-21-1219

View this article at: https://dx.doi.org/10.21037/apm-21-1219 


\section{Introduction}

Esophageal carcinoma (EC) is a clinically high incidence of gastrointestinal tumors, and China is a high-incidence area of such diseases (1). Related survey results have showed that, in 2014, the incidence of EC in China was $0.22 \%$, with an average of 150,000 deaths per year (2). With the continuous development of minimally invasive techniques in recent years, minimally invasive radical resection of $\mathrm{EC}$ is an important treatment for this disease. Minimally invasive radical surgery (MIS) mainly includes three steps: (I) free thoracic esophagus with thoracic lymph node dissection under laparoscopic; (II) free stomach, make tubular stomach and abdominal lymph node dissection under laparoscopic; (III) small left neck incision for tubular gastroesophageal anastomosis; due to the global medical equipment, technology, and treatment concepts vary from region to region. Postoperative patients with $\mathrm{EC}$ are prone to malnutrition. Studies have shown that the incidence of malnutrition after EC surgery reaches $60-80 \%$, and deaths due to malnutrition account for about $22 \%(3,4)$. Patients with EC need at least 3 months to establish a new dietary pattern after surgery, so short-term enteral nutrition is of great significance. Family enteral nutrition (FEN) is to provide home enteral nutrition support after the patient is discharged from the hospital under the guidance of a professional nutrition support group (5). Chen et al. found that in order to optimize the efficacy of EN, a home EN should last no less than eight weeks after discharge, and persistent EN can help improve the immune function of elderly patients undergoing radical EC surgery (6). In the present study, FEN was applied to elderly patients with EC after MIS, and body mass index (BMI), nutritional risk screening, nutritional status, and the incidence of complications were examined.

We present the following article in accordance with the CONSORT reporting checklist (available at https://dx.doi. org/10.21037/apm-21-1219).

\section{Methods}

\section{Patient selection}

A total of 106 elderly patients with EC who underwent MIS at the 904th Hospital of the Joint Service Support Center of the Chinese People's Liberation Army and Taixing People's Hospital from January 2017 to July 2019 were selected and using the random number table method, patients were divided into an intervention group and control

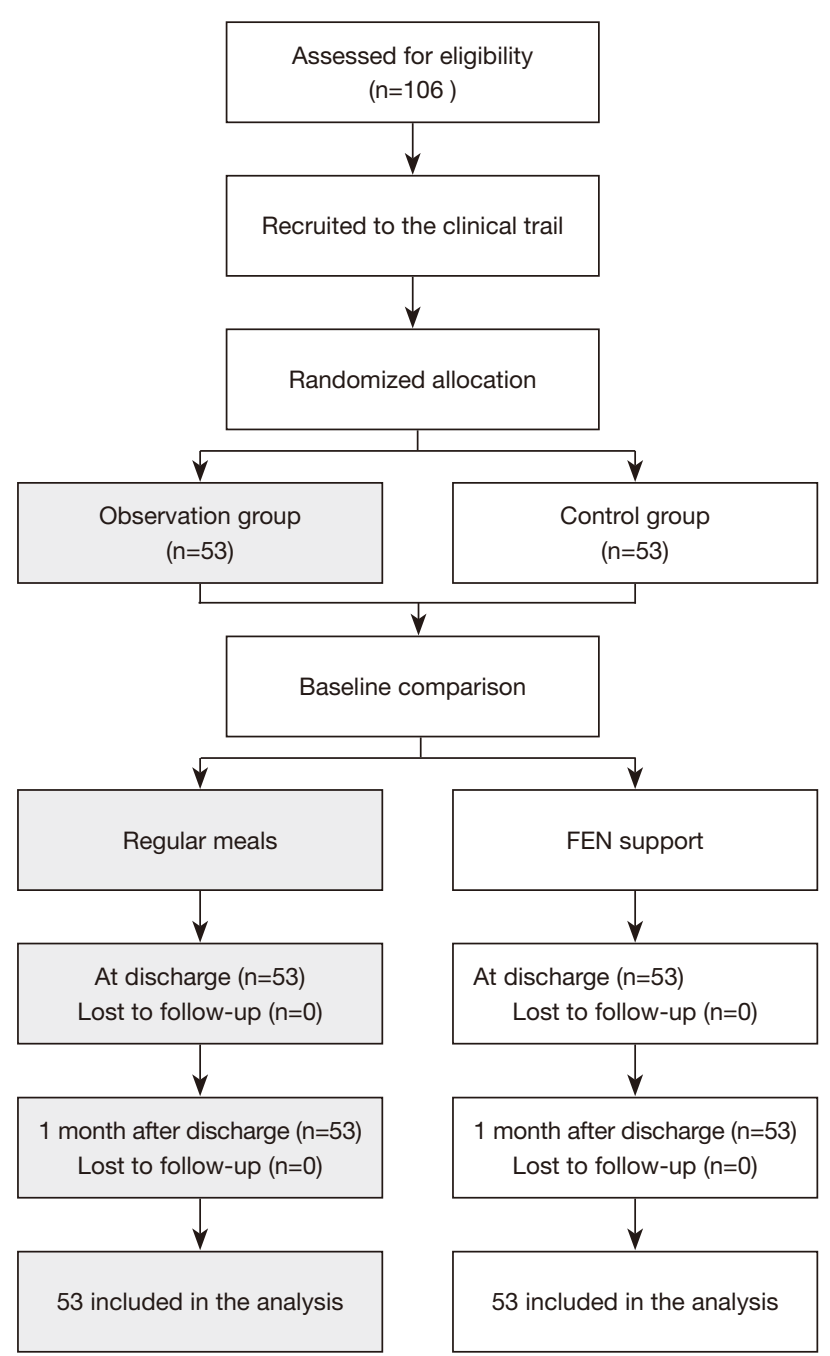

Figure 1 Flowchart of participants. FEN, family enteral nutrition.

group according to the ratio of 1:1 (Figure 1). There were 53 cases in each group. Patients met the following criteria to be eligible to participate in the present study: (I) diagnosed with EC through clinical examination, gastroscopy, and pathological examination; (II) aged $\geq 60$ years old; (III) underwent minimally invasive radical resection of EC; (IV) had a clear consciousness; (V) did not require radiotherapy and chemotherapy for 3 months after surgery; and (VI) provided signed informed consent. Patients were excluded from the study if they met any of the following exclusion criteria: (I) severe cognitive dysfunction; (II) language communication impairment; (III) severe complications after surgery; (IV) unable to tolerate immune enteral nutrition preparations; (V) poor treatment compliance; (VI) withdrawal from the study halfway through. The study was 
approved by the Ethics Committee of the 904th Hospital of the Joint Service Support Center of the Chinese People's Liberation Army and Taixing People's Hospital, and was conducted in accordance with the Declaration of Helsinki (as revised in 2013). All patients provided informed consent.

\section{Interventions}

The patients in the control group were given regular meals after they were discharged from hospital. Intervention staff undertook a nutritional assessment of patients before they were discharged from hospital and instructed them to consume ordinary meals. The patients underwent home rehabilitation and were provided with dietary health manuals. Home nutrition guidance included the following: (I) patients were encouraged to eat steamed eggs, millet porridge, mashed potatoes, pumpkin porridge, and other easily digestible foods; (II) the diet changed from thin to thick, and patients were encouraged to 5-8 small meals daily; (III) patients were instructed not to speak or communicate when eating, to chew slowly, and avoid eating hard and indigestible foods, such as rice cakes and bamboo shoots; and (IV) Instructed patients not to smoke, drink alcohol, and not eat fried and overnight foods. The patient could gradually transition to soft food 1 month after surgery. After the patient was discharged from hospital, the intervention staff followed up by telephone once a month to discuss the patient's daily diet and changes in their condition. The jejunostomy tube was removed during the hospital review 1 month later.

The observation group underwent FEN support based on the intervention of the control group. After being discharged from hospital, patients were provided with regular home dietary guidance, as well as enteral nutritional emulsion (TPF-T; Fresenius Kabi Huarui Pharmaceutical, National Medicine Standard H20040722) tube feeding. The dietitian determined the patient's daily intake based on their basic information and daily activity. Specialist physicians, nutritionists, and specialist nurses formed a FEN support group. The FEN support team evaluated whether the patient and their family members could undertake FEN 3 days before discharge and provided guidance on enteral nutrition and related knowledge training. The main content of the training included the infusion of home nutrient solution, the routine nursing operation of the catheter, the nursing of jejunostomy, and the prevention of complications and the coping methods. Carried out the assessment before the patient is discharged from the hospital. After passing the assessment, a FEN treatment card was issued, and a patient file was established to record their general information, condition, nutritional status, and contact information. After the patient was discharged from the hospital, the patient was gavaged with $200-500 \mathrm{~mL}$ of TPF-T daily, which slowly injected through the jejunostomy tube. Nutritional support was given 3-6 times a day. The interval between each time needed to be greater than $2 \mathrm{~h}$. The temperature of the nutrient solution was kept at $35^{\circ} \mathrm{C}$. The pipeline was flushed with warm water before and after the infusion. The FEN support team developed a follow-up plan. The FEN support team conducted telephone follow-up with patients once a week. The follow-up content included the patient's diet, physical condition, self-monitoring and enteral nutrition infusion. The FEN support team also needed to ask and record whether the patient has diarrhea, jejunostomy blockage, etc. after the infusion, and encourage the patient to adhere to FEN. One month after the operation, the follow-up frequency was changed to monthly. The jejunostomy tube was removed during the patient's hospital review 1 month later.

\section{Evaluation indexes}

\section{BMI}

The BMI of patients in both groups were recorded at the time of discharge and 1 month after discharge as follows: $\mathrm{BMI}=$ weight $(\mathrm{kg}) / \mathrm{height}\left(\mathrm{m}^{2}\right)$.

\section{Nutritional risk screening}

The European Nutritional Risk Screening 2002 (NRS 2002) was used to assess the nutritional risk of the two groups at discharge and 1 month after discharge. NRS 2002 $\geq 3$ points was classified as high nutritional risk, and NRS $2002<3$ points was considered to have no nutritional risk (7).

\section{Nutritional status}

Levels of hemoglobin, serum albumin, serum total protein, transferrin, and serum prealbumin upon discharge and 1 month after discharge were recorded.

\section{Complications}

The incidence of complications, including nausea and vomiting, diarrhea, and abdominal distension, were recorded 1 month after discharge in the two groups.

\section{Statistical analyses}

The statistical analysis in the present study was performed 
using SPSS version 20.0 (SPSS, Chicago, IL, USA). BMI and nutritional status of the two groups at discharge and 1 month after discharge were expressed as mean \pm standard deviation $(\bar{x} \pm \mathrm{s})$ and compared using two-tailed $t$-tests. Nutrition risk screening and complications of the two groups at discharge and 1 month after discharge were expressed by rates and percentages, and compared using the $\chi^{2}$-test or rank-sum test. $\mathrm{P}<0.05$ was considered statistically significant.

\section{Results}

\section{General information}

The present study comprised 106 patients with minimally invasive radical resection of EC. The control group included 41 males and 12 females, aged 61-78 years, with an average age of $67.79 \pm 7.24$ years. In terms of education, 11 cases had an education of elementary school and below, 20 cases had a junior high school education, 14 cases had a high school or technical secondary school education, and 8 cases had a junior college and above education. In terms of disease stage, 24 cases were stage I and 29 cases were stage II. Thirtytwo cases were married and 21 cases were single/divorced/ widowed. In relation to family income, 6 cases earned $<4,000$ yuan per month, 28 cases earned $4,000-8,000$ yuan per month, and 19 cases earned $>8,000$ yuan per month. The observation group comprised 43 males and 10 females, aged $62-77$ years, with an average age of $67.83 \pm 7.17$ years. In terms of level of education, 12 cases had an education of elementary school and below, 18 cases had a junior high school education, 13 cases had a high school or technical secondary school education, 10 cases had a junior college and above education. In terms of disease stage, 25 cases were stage I and 28 cases were stage II. Thirty-one cases were married and 22 cases were unmarried/divorced/widowed. In relation to family income, 6 cases earned $<4,000$ yuan per month, 30 cases earned 4,000-8,000 yuan per month, and 17 cases earned $>8,000$ yuan per month.

There were no statistically significant differences between the two groups of patients in terms of general data, such as sex, age, education level, disease stages, marital status, or family income $(\mathrm{P}>0.05)$ (Table 1).

\section{BMI}

At discharge, the BMI of the observation group was $20.38 \pm 2.15 \mathrm{~kg} / \mathrm{m}^{2}$, and it was $20.45 \pm 2.24 \mathrm{~kg} / \mathrm{m}^{2}$ for the control group. There was no statistical difference between the two groups $(\mathrm{t}=0.164, \mathrm{P}=0.870)$. One month after discharge, the BMI of the observation group was $21.47 \pm 2.26 \mathrm{~kg} / \mathrm{m}^{2}$, and it was $20.21 \pm 2.02 \mathrm{~kg} / \mathrm{m}^{2}$ for the control group. The difference between the two groups was statistically significant $(t=3.026, P=0.003)$. The BMI of the observation group was higher 1 month after discharge than that at discharge $(t=2.544, \mathrm{P}=0.012$ ), while the $\mathrm{BMI}$ of the control group was not significantly higher 1 month after discharge than that at discharge $(\mathrm{t}=0.580, \mathrm{P}=0.564)$ (Table 2).

\section{Nutritional risk screening}

At discharge, all patients in the observation group and the control group were at nutritional risk. One month after being discharged from the hospital, there were 24 patients in the observation group who were at nutritional risk, which was significantly lower than the 35 patients in the control group $\left(\chi^{2}=4.625, \mathrm{P}=0.032\right)$ (Table 3$)$.

\section{Nutritional status}

At discharge, there were no significant differences in levels of hemoglobin, serum albumin, total serum protein, transferrin, and serum prealbumin in the observation group compared with the control group $(\mathrm{t}=0.204,0.131$, $0.343,0.118,0.017 ; \mathrm{P}=0.839,0.896,0.733,0.906,0.987$ ). One month after discharge, levels of hemoglobin, serum albumin, total serum protein, transferrin, and serum prealbumin in the observation group were higher than those in the control group, and the differences were statistically significant ( $\mathrm{t}=8.925,7.851,8.697,8.110,4.644 ; \mathrm{P}=0.000$, 0.000, 0.000, 0.000, 0.000) (Table 4).

\section{Complications}

One month after discharge, the observation group had 2 cases of nausea and vomiting and 1 case of abdominal distension; the complication rate was $5.66 \%$. The control group had 3 cases of nausea and vomiting, 2 cases of diarrhea, and 2 cases of abdominal distension; the complication rate was $13.21 \%$. The incidence of complications in the two groups was not statistically significant $\left(\chi^{2}=1.767, \mathrm{P}=0.184\right)$ (Table 5).

\section{Discussion}

Enteral nutrition support for patients with EC is mainly used in the perioperative period, and its effect has been 
Table 1 Comparison of general information between the two groups

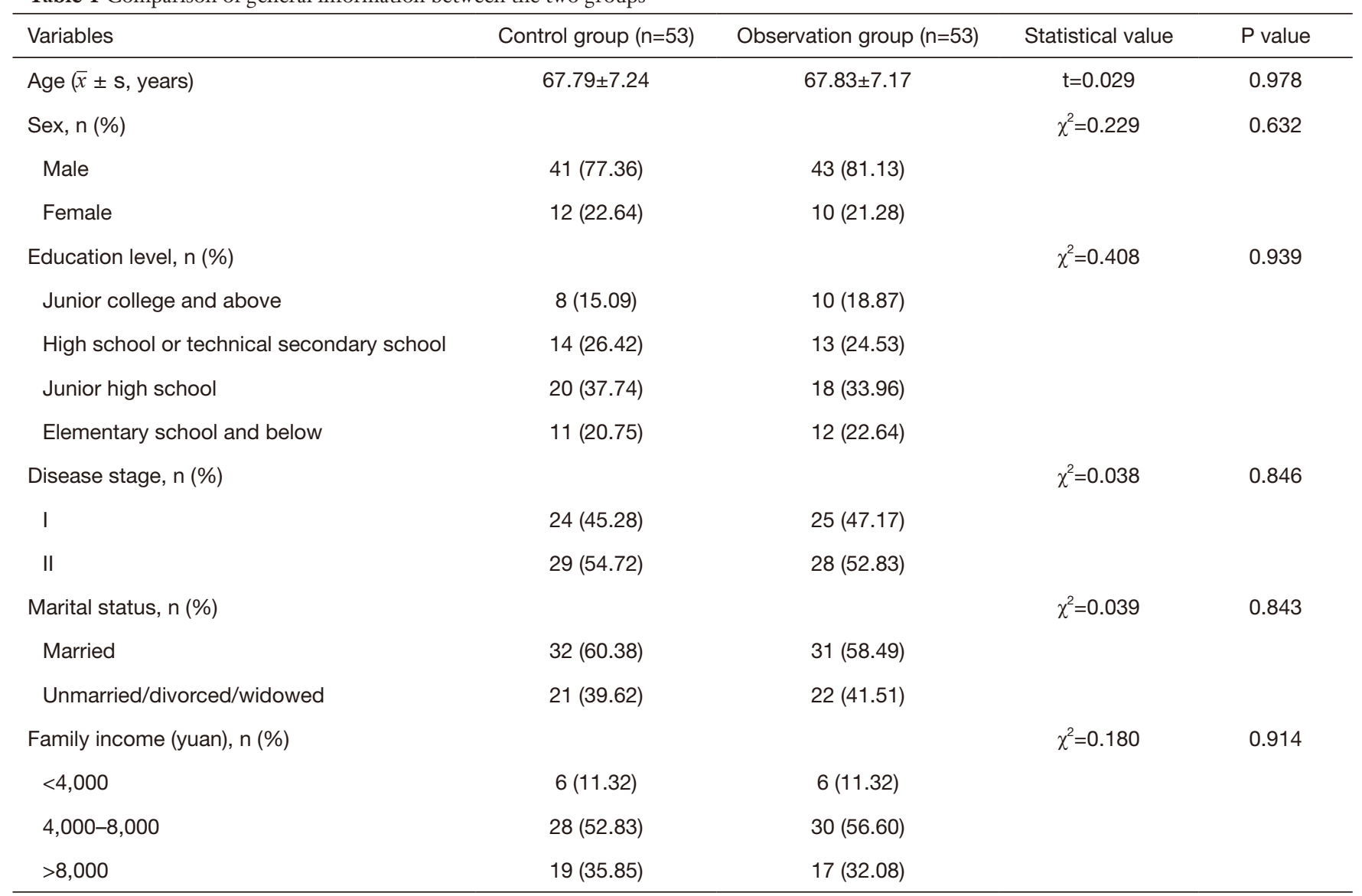

Table 2 Comparison of BMI between the two groups at discharge and 1 month after discharge $\left(\bar{x} \pm \mathrm{s}, \mathrm{kg} / \mathrm{m}^{2}\right)$

\begin{tabular}{|c|c|c|c|c|}
\hline Group & At discharge & 1 month after discharge & $\mathrm{t}$ value & $P$ value \\
\hline Control group $(n=53)$ & $20.45 \pm 2.24$ & $20.21 \pm 2.02$ & 0.580 & 0.564 \\
\hline $\mathrm{t}$ value & 0.164 & 3.026 & - & - \\
\hline$P$ value & 0.870 & 0.003 & - & - \\
\hline
\end{tabular}

BMI, body mass index.

widely recognized in clinical practice (8). However, in China, due to factors, such as limited bed numbers and high medical expenses, patients usually stop enteral nutrition after taking a semi-liquid diet without serious complications, and switch to oral diet and leave the hospital to go home to recuperate. Following EC surgery, gastric capacity is significantly reduced, and most patients can experience satiety and anorexia (9), and conventional home meals cannot meet their nutritional needs. Therefore, postoperative patients with EC would experience weight loss, body protein exhaustion, reduced immunity, and develop complications, such as infection, which significantly increase the probability of recurrence and adversely affect their prognosis $(10,11)$. Continuous enteral nutrition for patients after EC surgery is vital in order for them to meet their nutritional needs and improve their immunity. Compared to given regular meals, enteral nutrition support can maintain the normal physiological function of the 
Table 3 Comparison of nutritional risk screening between the two groups at discharge and 1 month after discharge

\begin{tabular}{|c|c|c|c|}
\hline Time & Group & At nutritional risk (NRS $2002 \geq 3$ points), $n$ (\%) & No nutritional risk (NRS $2002<3$ points), n (\%) \\
\hline \multirow{3}{*}{ At discharge } & Observation group $(n=53)$ & $53(100.00)$ & $0(0.00)$ \\
\hline & $\chi^{2}$ value & & - \\
\hline & $P$ value & & - \\
\hline \multirow{3}{*}{$\begin{array}{l}1 \text { month after } \\
\text { discharge }\end{array}$} & Observation group $(n=53)$ & $24(45.28)$ & $29(54.72)$ \\
\hline & $\chi^{2}$ value & \multicolumn{2}{|c|}{4.625} \\
\hline & $\mathrm{P}$ value & \multicolumn{2}{|c|}{0.032} \\
\hline
\end{tabular}

NRS 2002, Nutritional Risk Screening 2002.

Table 4 Comparison of nutritional status of the two groups at discharge and 1 month after discharge $(\bar{x} \pm \mathrm{s})$

\begin{tabular}{|c|c|c|c|c|c|c|}
\hline Time & Group & $\begin{array}{l}\text { Hemoglobin } \\
\qquad(\mathrm{g} / \mathrm{L})\end{array}$ & $\begin{array}{l}\text { Serum albumin } \\
(g / L)\end{array}$ & $\begin{array}{l}\text { Serum total protein } \\
\qquad(g / L)\end{array}$ & $\begin{array}{c}\text { Transferrin } \\
(\mathrm{g} / \mathrm{L})\end{array}$ & $\begin{array}{l}\text { Serum prealbumin } \\
(\mathrm{mg} / \mathrm{L})\end{array}$ \\
\hline \multirow[t]{3}{*}{ At discharge } & Control group $(n=53)$ & $10.83 \pm 1.24$ & $36.38 \pm 3.01$ & $65.17 \pm 5.43$ & $1.91 \pm 0.42$ & $186.27 \pm 53.75$ \\
\hline & Observation group $(n=53)$ & $10.78 \pm 1.28$ & $36.46 \pm 3.25$ & $65.53 \pm 5.39$ & $1.90 \pm 0.45$ & $186.09 \pm 55.82$ \\
\hline & t value & 0.204 & 0.131 & 0.343 & 0.118 & 0.017 \\
\hline \multirow{3}{*}{$\begin{array}{l}1 \text { month after } \\
\text { discharge }\end{array}$} & Observation group $(n=53)$ & $12.94 \pm 1.34$ & $41.88 \pm 2.75$ & $72.51 \pm 4.72$ & $2.47 \pm 0.41$ & $216.59 \pm 58.83$ \\
\hline & t value & 8.925 & 7.851 & 8.697 & 8.110 & 4.644 \\
\hline & $P$ value & 0.000 & 0.000 & 0.000 & 0.000 & 0.000 \\
\hline
\end{tabular}

Table 5 Comparison of complications between the two groups 1 month after discharge

\begin{tabular}{lcccc}
\hline Group & Nausea and vomiting, $\mathrm{n}(\%)$ & Diarrhea, $\mathrm{n}(\%)$ & Abdominal distension, $\mathrm{n}(\%)$ & Incidence of complications, $\mathrm{n}(\%)$ \\
\hline Control group $(\mathrm{n}=53)$ & $3(5.66)$ & $2(3.77)$ & $2(3.77)$ & $7(13.21)$ \\
Observation group $(\mathrm{n}=53)$ & $2(3.77)$ & $0(0.00)$ & $1(1.89)$ & $3(5.66)$ \\
$\chi^{2}$ value & - & - & - & 1.767 \\
P value & - & - & - & 0.184 \\
\hline
\end{tabular}

intestine, with high safety and wide selection of enteral nutrition agents (12). Enteral nutrition can more effectively improve the nutritional status of patients with esophageal cancer after operation, so as to protect the intestinal mucosal function and enhance gastrointestinal motility (13).

The main manifestation of malnutrition in patients with esophageal cancer is protein-energy-deficient malnutrition, and hemoglobin and serum albumin in the observation indicators are important indicators for evaluating the nutritional status of patients $(14,15)$. The results of the present study indicated that the observation group's BMI was higher than of the control group 1 month after discharge, and the observation group's serum total protein, hemoglobin, transferrin, serum prealbumin, and serum albumin levels were significantly higher than the control group after the intervention group $(\mathrm{P}<0.05)$. The findings 
indicated that FEN support could effectively improve the level of laboratory nutrition indicators among elderly patients with EC after MIS, and improve their BMI. The main aims of the present study were as follows. First, as a tumor-specific immune enteral nutrition preparation, TPF-T is rich in protein, energy, and fat, and has low carbohydrates $(16,17)$. Second, $\omega-3$ unsaturated fatty acids are added to TPF-T, and have better gastrointestinal tolerance than conventional enteral nutrition. TPF-T does not only promote the production of protein in the patient's body and improve nutritional status, but also helps the patient maintains body fat content in a short period of time and improves the body's immunity and cellular immunosuppressive state caused by surgical trauma $(18,19)$.

As a nutritional risk screening tool commonly used in clinical practice, NRS 2002 can objectively reflect the nutritional risk status of patients and help medical staff predict the clinical outcome of patients. An NRS 2002 score of $\geq 3$ indicates that the patient is at high risk of malnutrition, and treatment and prognosis of primary disease will have a serious negative impact $(20,21)$. The results of the present study indicated that the proportion of NRS 2002 scores $\geq 3$ in the observation group was significantly higher than that in the control group $(\mathrm{P}<0.05)$. FEN after discharge from the hospital among elderly patients with EC following MIS could effectively improve patients' feeding and nutritional status, and reduce the risk of malnutrition $(22,23)$.

The elderly patients in the observation group did not have intestinal fistula clogging or detubation, and no patients had diarrhea. This could be due to the enteral nutrition support received during the hospitalization; therefore, improving patients' intestinal tolerance and resulting in better compliance after discharge.

\section{Conclusions}

FEN could improve the nutritional status of elderly patients with EC after MIS, and reduce the risk of postoperative malnutrition and the incidence of complications.

\section{Acknowledgments}

Funding: None.

\section{Footnote}

Reporting Checklist: The authors have completed the
CONSORT reporting checklist. Available at https://dx.doi. org/10.21037/apm-21-1219

Trial Protocol: Available at https://dx.doi.org/10.21037/apm21-1219

Data Sharing Statement: Available at https://dx.doi. org/10.21037/apm-21-1219

Conflicts of Interest: All authors have completed the ICMJE uniform disclosure form (available at https://dx.doi. org/10.21037/apm-21-1219). The authors have no conflicts of interest to declare.

Ethical Statement: The authors are accountable for all aspects of the work, including ensuring that questions related to the accuracy or integrity of any part of the work are appropriately investigated and resolved. The study was approved by the Ethics Committee of the 904th Hospital of the Joint Service Support Center of the Chinese People's Liberation Army and Taixing People's Hospital (No. KY201603), and conducted in accordance with the Declaration of Helsinki (as revised in 2013). All patients provided informed consent.

Open Access Statement: This is an Open Access article distributed in accordance with the Creative Commons Attribution-NonCommercial-NoDerivs 4.0 International License (CC BY-NC-ND 4.0), which permits the noncommercial replication and distribution of the article with the strict proviso that no changes or edits are made and the original work is properly cited (including links to both the formal publication through the relevant DOI and the license). See: https://creativecommons.org/licenses/by-nc-nd/4.0/.

\section{References}

1. Akiyama Y, Iwaya T, Endo F, et al. Effectiveness of intervention with a perioperative multidisciplinary support team for radical esophagectomy. Support Care Cancer 2017;25:3733-9.

2. Zhao H, Koyanagi K, Kato K, et al. Comparison of longterm outcomes between radical esophagectomy and definitive chemoradiotherapy in patients with clinical T1bN0M0 esophageal squamous cell carcinoma. J Thorac Dis 2019;11:4654-62.

3. Xie SP, Fan GH, Kang GJ, et al. Esophageal reconstruction with remnant stomach: a case report and review of 
literature. World J Gastroenterol 2013;19:3169-72.

4. Matsuda Y, Habu D, Lee S, et al. Enteral diet enriched with $\omega-3$ fatty acid improves oxygenation after thoracic esophagectomy for cancer: a randomized controlled trial. World J Surg 2017;41:1584-94.

5. Wang G, Liu BR, Hu Z. Combined endoscopiclaparoscopic radical esophagectomy and lymph node dissection for the treatment of esophageal squamous cell carcinoma. Gastrointest Endosc 2020;91:192-3.

6. Chen X, Zhao G, Zhu L. Home enteral nutrition for postoperative elderly patients with esophageal cancer. Ann Palliat Med 2021;10:278-84.

7. Kanda M, Koike M, Kodera Y. ASO author reflections: characteristics associated with nodal and distant recurrence after radical esophagectomy for squamous cell carcinoma of the thoracic esophagus. Ann Surg Oncol 2020;27:3206-7.

8. Jiang H, Hua R, Sun Y, et al. Risk factors for anastomotic complications after radical mckeown esophagectomy. Ann Thorac Surg 2020. [Epub ahead of print]. doi: 10.1016/ j.athoracsur.2020.09.019.

9. Shimakawa T, Asaka S, Sagawa M, et al. Nutritional screening before surgery for esophageal cancer - current status and evaluation results. Gan To Kagaku Ryoho 2014;41:1301-3.

10. Morimoto H, Fujiwara Y, Lee S, et al. Treatment results of neoadjuvant chemoradiotherapy followed by radical esophagectomy in patients with initially inoperable thoracic esophageal cancer. Jpn J Radiol 2018;36:23-9.

11. Udagawa H, Ueno M, Haruta S, et al. Re-evaluation of the role of thoracoscopic esophagectomy as a Japanese-style radical surgery. Esophagus 2017;14:165-70.

12. Gramlich L, Hurt RT, Jin J, et al. Home enteral nutrition: towards a standard of care. Nutrients 2018;10:1020.

13. Yoshida N, Baba Y, Shigaki H, et al. Preoperative nutritional assessment by controlling nutritional status (CONUT) is useful to estimate postoperative morbidity after esophagectomy for esophageal cancer. World J Surg 2016;40:1910-7.

14. Fu X, Liu Q, Luo K, et al. Lymph node station ratio: Revised nodal category for resected esophageal squamous cell carcinoma patients. J Surg Oncol 2017;116:939-46.
15. Oya H, Koike M, Iwata N, et al. Feeding duodenostomy decreases the incidence of mechanical obstruction after radical esophageal cancer surgery. World J Surg 2015;39:1105-10.

16. Chen QJ, Ou L, Li K, et al. Meta-analysis of the relationship between Dietary Inflammatory Index and esophageal cancer risk. Medicine (Baltimore) 2020;99:e23539.

17. Pan F, Xu X, Zhang LL, et al. Dietary riboflavin deficiency induces genomic instability of esophageal squamous cells that is associated with gut microbiota dysbiosis in rats. Food Funct 2020;11:10070-83. Erratum in: Food Funct 2020.

18. Ma L, Luo GY, Ren YF, et al. Concurrent chemoradiotherapy combined with enteral nutrition support: a radical treatment strategy for esophageal squamous cell carcinoma patients with malignant fistulae. Chin J Cancer 2017;36:8.

19. Liu L, Wang YC, Liu QW, et al. Home enteral nutrition after esophagectomy for esophageal cancer: a systematic review and meta-analysis. Medicine (Baltimore) 2020;99:e21988.

20. Tan Z, Zhang M, Han Q, et al. A novel blood tool of cancer prognosis in esophageal squamous cell carcinoma: the Fibrinogen/Albumin Ratio. J Cancer 2017;8:1025-9.

21. Takagi K, Buettner S, Ijzermans JNM, et al. Systematic Review on the Controlling Nutritional Status (CONUT) score in patients undergoing esophagectomy for esophageal cancer. Anticancer Res 2020;40:5343-9.

22. Dong J, Zhang W, Zhang T, et al. Baseline nutritional status could be a predictor for radiation esophagitis in esophageal cancer patients undergoing radiotherapy. Ann Transl Med 2020;8:1148.

23. Cheng C, Ho RTH, Guo Y, et al. Development and feasibility of a mobile health-supported comprehensive intervention model (CIMmH) for improving the quality of life of patients with esophageal cancer after esophagectomy: prospective, single-arm, nonrandomized pilot study. J Med Internet Res 2020;22:e18946.

(English Language Editor: R. Scott)
Cite this article as: Chen T, Jiang W, He G. Effect of family enteral nutrition on nutritional status in elderly patients with esophageal carcinoma after minimally invasive radical surgery: a randomized trial. Ann Palliat Med 2021;10(6):6760-6767. doi: 10.21037/apm-21-1219 